\title{
Navigating the Problems with Early Systematic Reviews on Emerging Diseases: The Case of Coronavirus Disease 2019 (COVID-19) in Pregnancy.
}

\author{
Xue Ling Ang ${ }^{1}$, Sonali Prashant Chonkar², Monica Chua ${ }^{2}$, Suzanna Sulaiman $^{2}$, and Jill \\ $\mathrm{Lee}^{2}$ \\ ${ }^{1}$ NUS Yong Loo Lin School of Medicine \\ ${ }^{2}$ KK Women's and Children's Hospital
}

June 3, 2020

It has been six months since the outbreak of the Coronavirus Disease 2019 (COVID-19) and despite ongoing research efforts, much of it still remains uncertain. Since December 2019 when the infection was first identified, more than 16000 MEDLINE-indexed papers have been published surrounding this subject matter. It is of utmost importance to learn about COVID-19 infection and its potential effects on pregnancy and perinatal outcomes, in order to guide obstetrical management during this current disease outbreak. As such, many obstetric units have been expeditiously publishing data from their studies in an attempt to rapidly disseminate information on COVID-19 in pregnancy. To date, most of these publications have been in the form of case reports and case series. While these reports provide valuable information which have helped to guide early management in this rapidly evolving global pandemic, they are not ranked highly in the traditional hierarchy of evidence. [1]

Case reports are known to be at risk of publication bias and may not be suitable for statistical inferences. Case series typically lack controls, and are prone to bias. Furthermore, inconsistency across reporting and small sample size, make it difficult for conclusive inferences to be drawn. This may not be representative of the larger global situation. Thus, findings from these studies may not be widely applicable to larger populations of patients. [2] As we await better quality evidence from large laboratories and population databases, current systematic reviews and management guidelines have developed their recommendations based on findings from studies done during previous disease outbreak (e.g. Severe Acute Respiratory Syndrome (SARS) Coronavirus) and personal experiences with COVID-19, in addition to the current available literature on COVID-19. This has led to discrepancies in recommendations especially in areas with scarce data. This commentary aims to highlight some of the potential problems and limitations one may encounter when reviewing the existing literature.

In addition to the limitations in the study design of case reports and series, the problem of duplicate reporting needs to be addressed. Not only does duplicate reporting of the same patient overload available medical information, it also overemphasizes findings. This will in turn affect the accuracy of all subsequent data analyses and preclude valid systematic reviews. Therefore, it is of paramount importance to screen for overlapping cases before performing analysis.

This process has been challenging as some studies may not have clearly indicated if their patient series includes patients who may have been included in other published papers. This is especially of concern in areas where care or isolation facilities for COVID-19 are limited and patients may transfer between hospitals 
resulting in such cases being reported by both the admitting and receiving hospitals. Case studies may report on different aspects of the same case, rendering efforts to screen for duplicates by direct comparison of clinical characteristics and outcomes impossible. Some investigators have unfortunately reported failed attempts of contacting the corresponding author to identify the actual source of cases. [3]

A strict and rigorous system to screen for duplicates needs to be in place when performing a systematic review. Special attention should be paid to studies originating from countries with high COVID-19 related research output as risk of case duplication could be higher. Identifying the roles of the various healthcare institutions within the same country might be useful in identifying the admitting and receiving hospitals and thus, the possibility of overlapping cases. For studies derived from the same institution, direct comparison of data should be undertaken where applicable.

Apart from studies done during the peak of the COVID-19 outbreak in Hubei province, where patients were diagnosed with COVID-19 based on clinical signs and symptoms, epidemiological history and typical chest computed tomography (CT) findings, all other studies included only patients with laboratory confirmed positive quantitative reverse transcriptase polymerase chain reaction (qRT-PCR) assay. However, the viral nucleic acid test has a false-negative rate of up to 30\%. [4] Furthermore, studies have reported asymptomatic carriers who were only incidentally picked up during universal screening. [5] This suggests that many asymptomatic cases of COVID-19 have likely been undiagnosed. Thus, current literature may have underreported the actual prevalence of COVID-19 amongst pregnant women as well as the associated COVID-19 pregnancy outcomes and complications.

Conversely, as the follow-up period for these studies have been short and most patients are in their third trimester of pregnancy, there might be an overestimation of risks such as preterm birth whilst underestimating longitudinal risks such as fetal growth restriction. [6] The increased risk of preterm birth may also be confounded by the fact that some of these deliveries were expedited to optimize the maternal condition. As the indication for delivery may not always be clearly specified in case reports, it is near-impossible to extrapolate data about the rate of spontaneous versus iatrogenic preterm birth. It is important to recognise that other factors such as stress and anxiety, especially prevalent during a time of pandemic, can also have detrimental impact on perinatal outcomes. Hence, the lack of comparable controls negates attributing these complications and outcomes solely to COVID-19 infection.

Existing case reports and case series mainly focus on the characteristics of COVID-19 infection in pregnant women and the potential effects on pregnancy outcomes. Information revolving other peripartum issues such as breastfeeding, possibility of vertical transmission and the need for postpartum isolation, remains relatively limited.

Systematic reviews need to follow a strict study protocol with clearly stated inclusion and exclusion criteria. As a result, a large proportion of other resources such as commentaries, opinions, expert reviews, and letters to editors are typically excluded. Important clinical observations and findings based on experiences or experts' own research regarding COVID-19 might not be captured in these reviews.

Additionally, it should be highlighted that the findings of a systematic review are only accurate and updated up to the point of submission for publication. However, in the face of an evolving disease, new information and literature are emerging rapidly. Accounting for the time required for processing, findings may not always be the most updated by the time of publication. If more than 16000 papers can be published within a 6month period, it is a massive challenge for both researchers and readers to make conclusive decisions based on a single systematic review. To date, there are at least 8 published systematic reviews on COVID-19 and pregnancy with varying conclusions. [3, 6-12]

While timely reporting of findings regarding COVID-19 is necessary and crucial to supplement our understanding of the infection and thus, guide clinical management, reviewers and guideline developers should bear in mind the limitations and interpret findings with caution. At present, the conclusions drawn from the current body of evidence may be at risk of bias. Establishment of rigorously governed national and international registries can help to address some of these issues. Although it is important to recognize the 
limitations of these studies and reviews, we are immensely grateful for the tremendous ongoing research efforts during this challenging time of a global pandemic. The quick dissemination of information inevitably helps clinicians to keep abreast of the latest developments, thereby guiding and updating clinical management as deemed appropriate. As we continue the fight against COVID-19, we look forward to more extensive research outcomes, contributing to a higher level of evidence.

\section{Disclosure of Interests}

None. Authors do not have any conflicts of interests.

\section{Contribution to authorship}

XLA: Preparation, manuscript original writing and editing. SPC, MSQC and SS: supervision, manuscript review and edit. JCSL: Conceptualisation, supervision, manuscript review and edit.

\section{Details of ethics approval}

Ethics approval not applicable for this commentary.

\section{Acknowledgements}

None.

\section{References}

1. Royal College of Obstetricians and Gynaecologists. Developing a Green-top Guideline. London RCOG, 2020 [Available from: https://www.rcog.org.uk/globalassets/documents/guidelines/rcog-guidelinedevelopment-guide.pdf.

2. Sayre JW, Toklu HZ, Ye F, Mazza J, Yale S. Case Reports, Case Series - From Clinical Practice to Evidence-Based Medicine in Graduate Medical Education. Cureus. 2017;9(8):e1546.

3. Juan J, Gil MM, Rong Z, Zhang Y, Yang H, Poon LC. Effects of coronavirus disease 2019 (COVID-19) on maternal, perinatal and neonatal outcomes: a systematic review. Ultrasound Obstet Gynecol. 2020.

4. Calda P BM, Fischerová D, Zikán M, Smetanová D, Machala L. Coronavirus infection and pregnancy (COVID-19). Opinion of the Czech Society for Ultrasound in Obstetrics and Gynecology. . Actual Gyn. 2020;12:17-9.

5. Breslin N, Baptiste C, Gyamfi-Bannerman C, Miller R, Martinez R, Bernstein K, et al. COVID-19 infection among asymptomatic and symptomatic pregnant women: Two weeks of confirmed presentations to an affiliated pair of New York City hospitals. Am J Obstet Gynecol MFM. 2020:100118.

6. Di Mascio D, Khalil A, Saccone G, Rizzo G, Buca D, Liberati M, et al. Outcome of Coronavirus spectrum infections (SARS, MERS, COVID 1 -19) during pregnancy: a systematic review and meta-analysis. Am J Obstet Gynecol MFM. 2020:100107.

7. Yang Z, Wang M, Zhu Z, Liu Y. Coronavirus disease 2019 (COVID-19) and pregnancy: a systematic review. J Matern Fetal Neonatal Med. 2020:1-4.

8. Zaigham M, Andersson O. Maternal and perinatal outcomes with COVID-19: A systematic review of 108 pregnancies. Acta Obstet Gynecol Scand. 2020.

9. Della Gatta AN, Rizzo R, Pilu G, Simonazzi G. Coronavirus disease 2019 during pregnancy: a systematic review of reported cases. Am J Obstet Gynecol. 2020.

10. Abdollahpour S, Khadivzadeh T. Improving the quality of care in pregnancy and childbirth with coronavirus (COVID-19): a systematic review. J Matern Fetal Neonatal Med. 2020:1-9.

11. Kasraeian M, Zare M, Vafaei H, Asadi N, Faraji A, Bazrafshan K, et al. COVID-19 pneumonia and pregnancy; a systematic review and meta-analysis. J Matern Fetal Neonatal Med. 2020:1-8. 
12. Muhidin S, Behboodi Moghadam Z, Vizheh M. Analysis of Maternal Coronavirus Infections and Neonates Born to Mothers with 2019-nCoV; a Systematic Review. Arch Acad Emerg Med. 2020;8(1):e49. 